\title{
Application of a partial-thickness human ex vivo skin culture model in cutaneous wound healing study
}

\author{
Wei Xu, Seok Jong Hong, Shengxian Jia, Yanan Zhao, Robert D Galiano and Thomas A Mustoe
}

A number of in vivo and ex vivo skin models have been applied to human wound healing studies. A reliable skin model, which recapitulates the features of human wound repair, is essential for the clinical and mechanical investigation of human cutaneous wound healing. Full-skin ex vivo culture systems have been used in wound healing studies. However, important structures of the skin, such as the differentiation of keratinocytes and epidermis-dermis junction, are poorly characterized in this model. This study aims to develop an optimized partial-thickness human ex vivo skin culture (HESC) model to maintain human skin characteristics in vitro. During our culture, the basal layer, suprabasal layer, and stratum granulosum layer of epidermis were preserved until day 8. Analyses of hemidesmosome proteins, bullous pemphigoid antigen 1 (BP180) and 2 (BP230), showed that the integrity of the basement membrane of the epidermis was well preserved in the HESC model. In contrast, an organotypic culture with human keratinocytes and fibroblasts failed to show an integrated basement membrane. Maintenance of skin structure by histological analysis and proliferation of epidermal keratinocytes by Ki67 staining were observed in our model for 12 days. Complete re-epithelialization of the wounding area was observed at day 6 post wounding when a superficial incisional wound was created. The expression of Ki-67 and keratin 6 , indicators of activated keratinocytes in epidermis, was significantly upregulated and new collagen synthesis was found in the dermis during the wound healing process. As control, we also used organotypic culture in studying the differentiation of the keratinocyte layers and incisional wound repair. It turned out that our model has advantage in these study fields. The results suggest that our HESC model retains important elements of in vivo skin and has significant advantages for the wound healing studies in vitro.

Laboratory Investigation (2012) 92, 584-599; doi:10.1038/labinvest.2011.184; published online 9 January 2012

KEYWORDS: collagen reproduction; human skin ex vivo culture; keratinocyte apoptosis; keratinocyte proliferation; keratinocyte differentiation; keratinocyte migration; wound healing

Wound closure is a complex process involving multiple factors in several dynamic phases. A successful experimental model will encapsulate each phase to better understand the mechanistic features of wound repair and to the development of biological therapeutics for clinical use. ${ }^{1}$ Currently, popular wound healing models can be classified as in vivo or in vitro with model selection dependent on the objectives and hypotheses of the investigation. An in vivo model utilizing animal subjects has the advantages of simulating wound healing that is most similar to clinical cases. For example, the host's vascular and immune systems, as well as the external environment, influence animal models like humans. Although mouse, ${ }^{2}$ rat $^{3}$ rabbit, ${ }^{4}$ and guinea pig $^{5}$ wound healing models are used to imitate human skin repair, these models are affected by physiological differences. For example, wounded mice and rats heal by contraction of the subcutaneous muscle (panniculus carnosus) instead of re-epithelialization as seen in humans. As this subcutaneous muscle layer does not exist in humans, contraction contributes to a different healing mechanism. ${ }^{6}$ Also, the in vivo animal model requires a number of replicates for each treatment condition to minimize the effects of intraspecies differences. The variation in anatomy, physiology, pathology, and stress experienced by animals can also alter research results. ${ }^{6}$

Unlike in vivo models, in vitro wound closure models, such as mono-layer keratinocyte culture, ${ }^{7,8}$ keratinocyte-fibroblast

Laboratory for Wound Healing Repair and Regenerative Medicine, Department of Surgery, Division of Plastic and Reconstructive Surgery, Northwestern University, Feinberg School of Medicine, Chicago, IL, USA

Correspondence: Dr TA Mustoe, MD, Department of Surgery, Division of Plastic and Reconstructive Surgery, Professor of Northwestern University, Feinberg School of Medicine, 303 E Chicago Avenue, Tarry 4-720, Chicago, IL 60611, USA.

E-mail: t-mustoe@northwestern.edu

Received 4 April 2011; revised 17 August 2011; accepted 27 September 2011 
co-culture, ${ }^{9,10}$ organotypic culture, ${ }^{11,12}$ and skin explants ${ }^{13-15}$ directly utilize human cells to simulate the living environment of human skin. Wound-healing studies can be carried out in each representational model. In vivo models have been applied to numerous studies related to cutaneous wound closure including response to stress, dose response interactions, and immune response. ${ }^{1,16}$ However, very few in vitro models can completely reproduce the physiology of human skin for wound repair studies. ${ }^{17}$ Lack of multidimensional growth makes the mono-layer keratinocyte culture a less robust tool for wound healing investigation. Despite the fact that both keratinocyte-fibroblast co-culture and organotypic culture have the characters of three-dimensional cell culture, their fidelity to in vivo is still limited. The main cell types for both models are keratinocytes and fibroblasts. Although this gives researchers an opportunity to study the interaction of these two types of cells, the surrounding environments of the cells are different than that of human skin. The matrix of these in vitro models has been simplified. Also, the role of the immune response (neutrophils, macrophages, dendritic cells, etc), an important part of the wound healing process, is negated and has no function in these systems. Furthermore, although the organotypic culture of keratinocytes and fibroblasts (OTC) introduces type I collagen or fibrin to mimic the dermal matrix, the phenotype of cultured fibroblasts in the collagen is still altered as the mechanical tension in the system are different than that of real skin. ${ }^{18}$

On the other hand, human full-skin ex vivo cultures contain other cellular elements of skin such as Langerhans cells, pigment cells, and nerve endings. Previous studies with human full-skin ex vivo culture systems have been employed in many studies related to human dermatology, such as functional study of epidermal growth factor (EGF) in psoriasis, ${ }^{19}$ expression of cytokines in psoriatic skin, ${ }^{20}$ epidermal Langerhans cell behaviors, ${ }^{21}$ and transgenic studies for immunotherapeutic purposes. ${ }^{22-25}$ The full-skin culture has also been used in the study of epidermis epithelialization, ${ }^{26}$ infection during wound healing, ${ }^{6}$ as well as tensile strength of skin wound healing. ${ }^{14}$

Several strategies have been previously used in maintaining the ex vivo full-skin culture. In these models, the thickness of the skin, the type of culture media, and even the platform to support the skin were different. For example, in the study of transfection and activation of human cutaneous dendritic cells, a partial-thickness skin culture was cultured on a sterile steel mesh with a supply of RPMI 1640 supplemented with $10 \%$ fetal bovine serum (FBS). ${ }^{22}$ In another model, to study wound infection ex vivo, a full-thickness skin raft was cultured on a stainless steel chamber surrounded by Dulbecco'smodified Eagle's medium (DMEM) containing 10\% FBS. Surprisingly limited characterization of the cultured skinsuch as basement membrane integrity, proliferation of keratinocytes, and duration of survival of skin-was described in these ex vivo studies. In particular, major unanswered questions are the time in culture that ex vivo cultures persist with respect to proliferation and apoptosis, and the ability to respond to injury. The aim of this study is to develop an optimized partial-thickness of human ex vivo skin culture (HESC) model to maintain human skin characteristics in vitro. Rather than the full-skin ex vivo culture model, HESC model includes epidermis and only a very thin layer of dermis. Both histological and biochemical analyses show that the properties of skin found in vivo were maintained in our HESC model. Re-epithelialization of incisional wounds was also found in our model. Furthermore, we compared our HESC model with a classic OTC model in both morphology and wound healing study. These results suggest that our HESC model retains important elements of in vivo skin and has significant advantages for the wound healing studies in vitro.

\section{MATERIALS AND METHODS Preparation of HESC Model}

Human skin was collected from elective abdominoplasties. All human tissues were obtained according to the guidelines set forth by Northwestern University and under a protocol approved by the Institutional Review Board of the Northwestern University. Subcutaneous fat was carefully removed with an industrial razor blade. Skin was disinfected with Betadine and $70 \%$ ethanol, and then fixed on a sterilized cutting board. A thin layer of skin with complete epidermis and partial-thickness dermis (Figure 1) was sliced and washed in Dulbecco's phosphate-buffered saline with penicillin-streptomycin (Invitrogen Life Technologies, Carlsbad, CA, USA). The skin tissue was put on the bottom of a nylon mesh cell strainer (BD Biosciences, San Jose, CA, USA) with the epidermis facing up. Eight sutures were applied to each piece of skin to fix the skin on the strainer. The cell strainer was then placed into a six-well plate (Figure 2). The cell culture medium consisted of DMEM (Mediatech, Herndon, VA, USA) supplemented with $10 \%$ FBS (Thermo Scientific HyClone, Logan, UT, USA) and penicillin-streptomycin. The skin graft was cultured in the liquid-air interphase and kept in the cell culture incubator at $37^{\circ} \mathrm{C}$ with $5 \% \mathrm{CO}_{2}$. The medium was changed every other day.

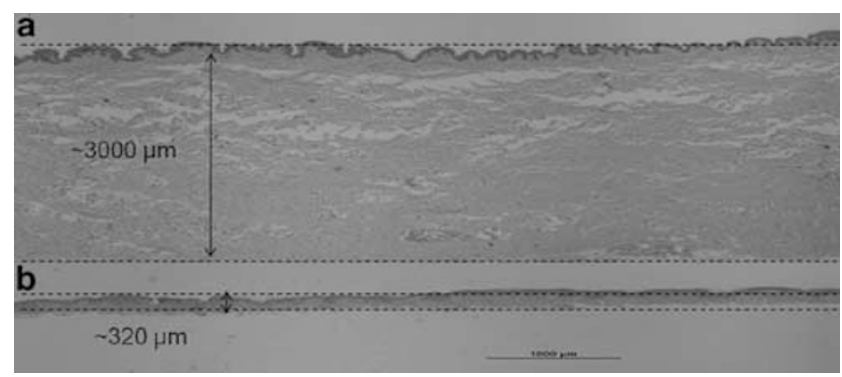

Figure 1 Comparison of full-thickness human skin to HESC. The normal full-thickness human skin (a) and cultured partial-thickness skin (b) have an average thickness of $3000 \mu \mathrm{m}$ and $320 \mu \mathrm{m}$, respectively. 


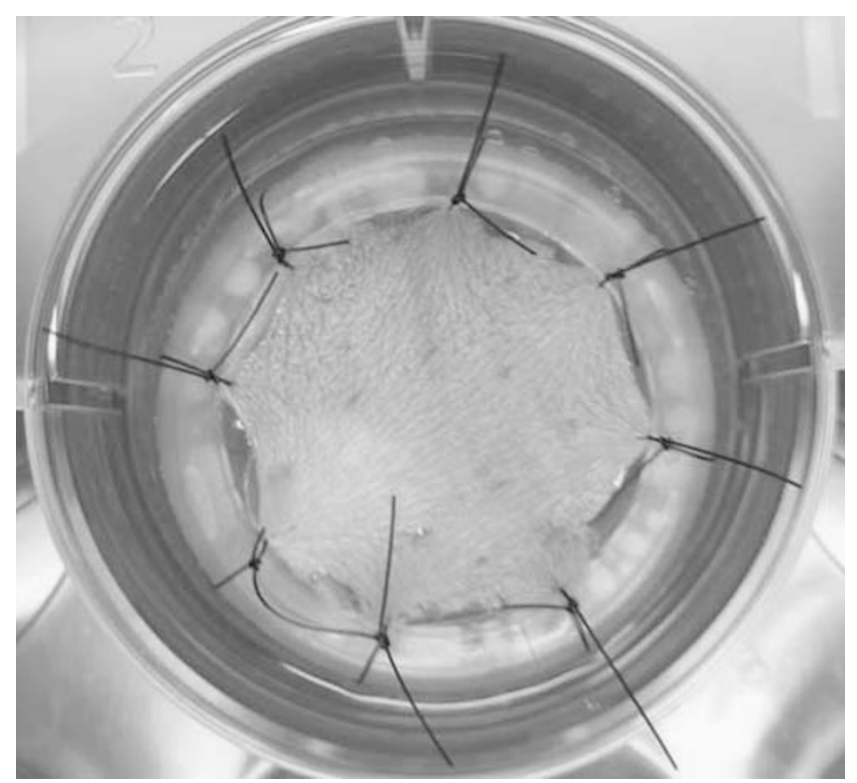

Figure 2 Partial-thickness human ex vivo skin culture. A small piece of skin graft was sutured on the bottom outside surface of a cell strainer.

\section{Preparation of OTC Model}

The human organotypic raft cultures were directly purchased from the Skin Disease Research Center (SDRC) at Northwestern University, Feinberg School of Medicine. Briefly, fibroblasts and liquid collagen mixture, containing 750000 human fibroblast cells were added into an insert sitting in a six-well plate. A fibroblast cell culture medium DMEM was added to submerge the plug and was incubated at $37^{\circ} \mathrm{C}$ for $24 \mathrm{~h}$. Then, one million keratinocytes were suspended with E-medium supplied by SDRC and plated on the top of each plug. The E-medium was also used to replace the DMEM surrounding the plug. E-medium contains 50:50 (v/v) DMEM and DMEM/F-12, E-cocktail mix $(18 \mu \mathrm{M}$ adenine, $500 \mathrm{ng} / \mathrm{ml}$ bovine pancreatic insulin, $500 \mathrm{ng} / \mathrm{ml}$ human Apotransferring, and $500 \mathrm{ng} / \mathrm{ml}$ triiodothyronine, $4 \mathrm{mM}$ L-glutamine, $0.4 \mu \mathrm{g} / \mathrm{ml}$ hydrocortisone, $10 \mathrm{ng} / \mathrm{ml}$ cholera toxin, $5 \mathrm{ng} / \mathrm{ml} \mathrm{EGF}$, 5\% FBS, and antibiotics). The plate was incubated at $37^{\circ} \mathrm{C}$ for $48 \mathrm{~h}$ then the E-medium was carefully removed from the top of each plug (lifting). The surrounding E-medium was changed every other day until the sample was harvested. The skin rafts were harvested at day 12 post lifting.

\section{Histological Structure of HESCs and OTCs}

The cultured skin grafts were harvested at day $0,2,4,10,12$, 14 , and immediately fixed with $10 \%$ formalin. The fixed tissues were then embedded in paraffin and sectioned with $5 \mu \mathrm{m}$ thickness. The tissue section were stained with hematoxylin \& eosin (H\&E) solutions and observed under Nikon Eclipse 50i light microscope with the images captured by Nikon Digital Sight DS-U1 camera system (Nikon, Melville, NY). The image process was performed by software NISElements BR 2.30 (Nikon).

\section{Detection of Keratinocyte Differentiation and Dermis- Epidermis Junction (DEJ) in HESCs and OTCs}

Rabbit anti-keratin 5 (K5, Abcam, Cambridge, MA, USA), mouse-anti-keratin 10 (K10, Dako, Carpinteria, CA, USA), and rabbit-anti-loricrin (Abcam) antibodies were used to identify the basal, spinous, and granular layers in the epidermis of HESCs and OTCs. Two hemidesmosome proteins, BP180 and BP230, were analyzed with the mouse-anti-BP180 and human-anti-BP230 (gifts from Dr Jonathan Jones) to detect the DEJ as well as the integrity of basement membrane of the epidermis. Alexa Fluor 488 or 594 (Invitrogen, Carlsbad, CA) conjugated antibodies were used after primary antibody treatment. Nuclei of the cells were stained by $4^{\prime}, 6$-diamidino-2-phenylindole. The signals were detected by fluorescent microscopy.

\section{Keratinocytes Apoptosis and Proliferation in HESCs}

The HESCs were harvested at day 0, 2, 4, 10, 12, and 14 . Tissues were immediately embedded in the optimal cutting temperature (O.C.T.) compound and snap frozen in liquid nitrogen. The embedded samples were then cross-sectioned with $10-\mu \mathrm{m}$ thickness. Apoptosis was assessed using the terminal deoxynucleotidyl transferase dUTP nick end labeling (TUNEL) system (Promega, Madison, WI) according to manufacturer's instructions. For the proliferation assay, mouse-anti-Ki67 antibody (BD Biosciences Pharmingen, San Diego, CA) was used. A biotinolyated goat-anti-mouse IgG was used as a secondary antibody. The signal was detected using the Vectastain Elite ABC system (Vector Labs, Burlingame, CA) and visualized using 3, $3^{\prime}$-diaminobenzidine. To determine the proliferation rate of the basal layer keratinocytes, the numbers of Ki67-positive cells in the basal layer keratinocytes were counted. To determine the apoptosis rate of keratinocytes, TUNEL staining positive cells were counted in the full depth of the epidermis. Five random fields per sample were analyzed from three samples and the rate was presented as mean \pm s.e.m. The significance of the difference between each two time points was estimated by ANOVA with post hoc Tukey's adjustment.

\section{Study of Re-Epithelialization on HESCs and OTCs}

The HESCs were prepared as described above and incubated with a medium in the cell culture incubator overnight. Thereafter, an incisional wound created by a scalpel cut completely through the epidermal layer and superficially nicked the dermis. Shallow perpendicular incisions were made evenly on the surface of the skin grafts with a maximum distance of $1 \mathrm{~mm}$ between wounds. The HESCs were then continuously cultured at the same conditions as described above and were harvested at day $0,5,7,9,12$, and 14 post wounding. The histological analysis was performed on the harvested samples with H\&E staining. In addition, picrosirius red (Direct red 80, Sigma-Aldrich, St. Louis, MO) staining was used to visualize the collagen reconstruction during wound healing. Anti-Ki-67 antibody was used to 
detect the induction of keratinocytes in wound beds. To detect the induction of the cytokeratin 6 (K6) expression in the epidermis during wound healing, the skin samples were embedded with O.C.T. and $10 \mu \mathrm{m}$ cross-sectional slides were made with frozen blocks. The immunofluorescent staining was performed on the slides with a mouse anti-K6 antibody (Abcam). The unwounded HESCs were used as controls.

To study re-epithelialization in the OTCs, incisions were created at day 7 post lift of the culture (consider as postoperative day 0 (POD0) for OTCs). The samples were harvested every other day until day 12 post lift (POD12) and analyzed as described above.

\section{RESULTS}

\section{The Structure of the Skin was Maintained During the Culture of HESCs}

Compared with the fresh human skin sample, which was processed within $4 \mathrm{~h}$ after collection from the patient (Figure 3a), the HESCs at day 4 (Figure 3c) and day 10 (Figure 3d) showed increase in epidermal thickness. The number of keratinocyte layers was estimated by the thickness of the epidermis $(\mu \mathrm{m})$ divided by the average size of keratinocytes $(\mu \mathrm{m} / \mathrm{cell})$ measured in each slide. Approximately 5 layers of keratinocytes were observed in the epidermal layer of the skin day 0 , whereas about 12 and 11 layers of keratinocytes were seen at day 2 and day 4 during ex vivo culture, respectively. At day 10 (Figure 3d) and day 12 (Figure 3e), the numbers of keratinocyte layers in the epidermis were decreased compared with day 2 and day 4 but still more than fresh skin at day 0 . Notably, the thickness of stratum corneum of the HESCs at day 10 and day 12 were increased compared with both the fresh skin and skins at day 2 and day 4. The structure of the skin explants at day 14 demonstrated significant differences from the fresh skin sample (Figure 3f). After 14 days in culture some detachment of epidermis from dermis was observed (data not shown). Also, the thickness of the epidermis at day 14 was dramatically decreased and only a few keratinocytes were seen in the epidermis.

Differentiation of keratinocytes in HESCs progressed in normal fashion until day 12 based on the immunofluoresent staining of K5, K10, and loricrin. Expression of K5, a basal layer keratinocyte marker, was detected from day 0 to day 12 during culture primarily near the basal layer (Figures 4a, c, e, and g). At day 12, K5 was expressed both in the basal and suprabasal layers although the signal was weak (Figure $4 \mathrm{~g}$ ). Though expressions of $\mathrm{K} 5$ were also observed in the OTCs, signals were found in all the layers of epidermis in contrast to HESCs (Figures 4i, k, m and o). K10 was consistently expressed in a spinous layer of ex vivo epidermis until day 12 (Figures $4 a, c$, e, and g). In contrast, the K10 was only expressed in the spinous layer of organotypic raft at POD0 (Figure 4i). No signal of K10 was detected at the rest of the time points in organotypic culture (Figures $4 \mathrm{k}, \mathrm{m}$ and $\mathrm{o}$ ). Both ex vivo culture (Figures $4 \mathrm{~b}, \mathrm{~d}, \mathrm{f}$, and $\mathrm{h}$ ) and organotypic culture (Figures $4 \mathrm{j}, \mathrm{l}, \mathrm{n}$, and $\mathrm{p}$ ) had stratum granulosum until day 12 , indicated by the terminal differentiation keratinocyte marker, loricrin, though the signals at day 12 were attenuated (Figures $4 \mathrm{~h}$ and $\mathrm{p}$ ).

In assessing the survival and structure of the explants, the integrity of the basement membrane of the epidermis was measured through the detection of hemidesmosome antigen BP180 and BP230. The signals of both BP180 (Figures 5a-d) and 230 (Figures $5 \mathrm{e}-\mathrm{h}$ ) were shown at all time points along the time course of the HESC. The continuous distribution of BP180 and BP230 between the epidermis and dermis suggested the integrity of the basement membrane in the skin graft. Even at day 21 when the epidermis of the cultured skin appeared to be disrupted, the basement membrane was still well maintained based on BP180 and 230 staining (Figures 5d and $\mathrm{h}$ ). As a control, expression of BP180 and 230 was also analyzed on cross-sections of organotypic skin culture. The dashed distribution of BP180 and 230 along the basal layer of epidermis indicates an incomplete basement membrane at POD3 in OTCs (Figures 6a and b).

\section{Epidermal Keratinocytes Proliferate during ex vivo Skin Culture}

Proliferating keratinocytes were mainly found along the basal layer in the HESC (Figure 7). Quantification of the proliferating keratinocytes in the basal layer showed a high proliferation profile in the fresh skin epidermis $(45.05 \pm 1.12 \%$, Figure $7 \mathrm{a})$. A decrease in the number of proliferating keratinocytes was shown as early as day 2 ( $32.52 \pm 1.47 \%$, Figure $7 \mathrm{~b})$. A significant drop of proliferating keratinocytes was seen at day $12(7.27 \pm 2.30 \%$, Figure $7 \mathrm{e})$ compared with day $4(26.13 \pm 4.81 \%$, Figure $7 \mathrm{c})$. No proliferating keratinocytes were detected at day 14 (Figure 7f). Both histological and biochemical studies suggested that the structure of skin is well maintained and epidermal keratinocytes proliferate in HESC. The differences of cell proliferation rates between day 0 and any of the other time points were all significant based on ANOVA $(P<0.01)$.

Apoptosis of keratinocytes was analyzed along the time course of the ex vivo skin culture as both proliferation of keratinocytes (Figure 7) and thickness of epidermis (Figure 3) were drastically decreased at day 14 . The freshly collected skin sample did not show any signal for apoptosis in keratinocytes (Figure 8a). Apoptotic keratinocytes were clearly observed at day 6 culture $(12.72 \pm 0.57 \%$, Figure $8 b)$. Apoptosis of keratinocytes was also observed at later time points, including day $12(22.00 \pm 1.16 \%$, Figure $8 \mathrm{c})$ and day $14(29.44 \% \pm 3.41 \%$, Figure $8 \mathrm{~d})$, and the distribution range of apoptotic cells were extended across the whole epidermal layer. The apoptosis rate at day 0 was significantly lower than other time points based on ANOVA $(P<0.01)$.

\section{Re-Epithelialization and New Collagen Synthesis were Found in the Incisional Wounds on HESCs}

We addressed the wound healing process in HESC by creating partial-thickness incisional wounds. It was clearly shown that 

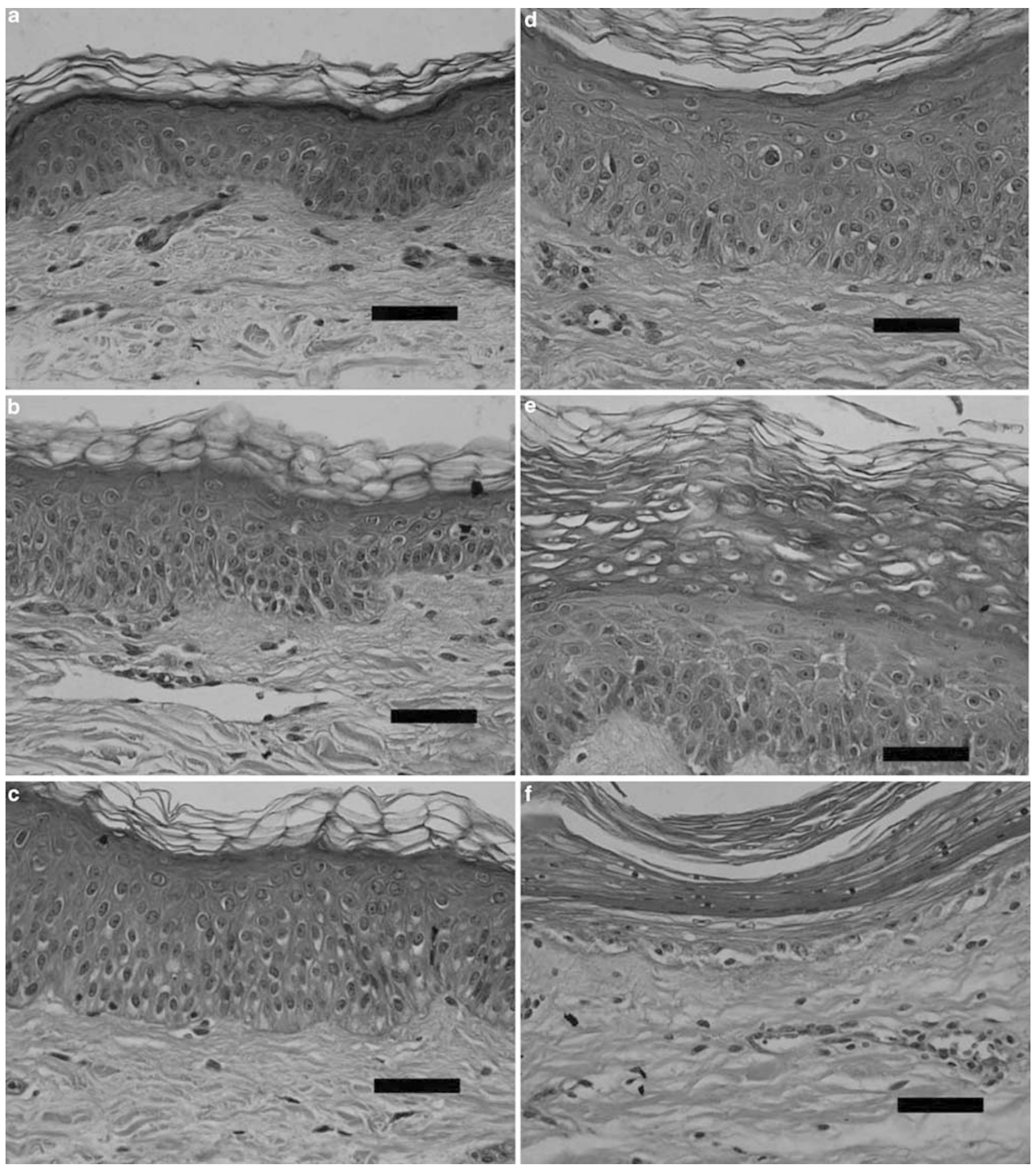

Figure 3 Morphological changes in the HESCs along the time course of the culture. Compared with control skin (a), the skin at day 2 (b) did not show any significant differences in appearance. Starting from day 4 (c), the epidermal layer of the skin graft appeared to be thicker than the control. The skin at day 10 (d) and day 12 (e) also had thicker epithelial layer than control, whereas the stratum corneum layer of the skin at day 12 was thicker than control. At day 14 (f), epidermal layer was significantly decreased in size compared with other time points. The length of the scale bar is $50 \mu \mathrm{m}$.

the incisions on the skin explants penetrated the full thickness of the epithelial layer and superficially nicked the dermis at day 0 (Figure 9a). The gap in the epidermis was still found at day 2 post wounding; however, the keratinocytes at the margins of the gap showed signs of migration (Figure 9b). The re-epithelialization of epidermis was completed on day 6 (Figure 9c). On day 8, the epidermis showed hyperplasic epithelium surrounding the original incision (Figure 9d). 
Ex vivo Skin Culture

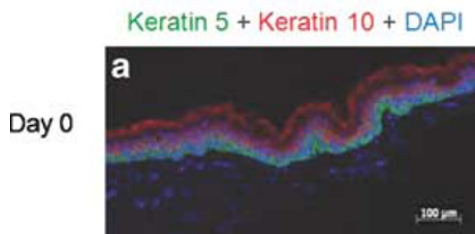

Day 4

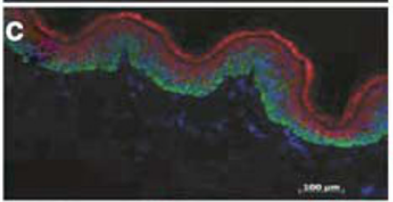

Day 8

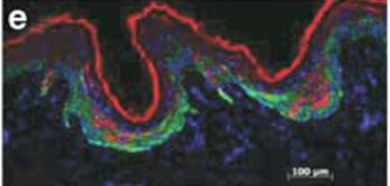

Day 12

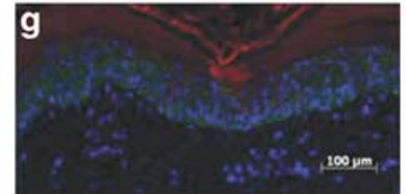

Loricrin+ Keratin $10+$ DAPI
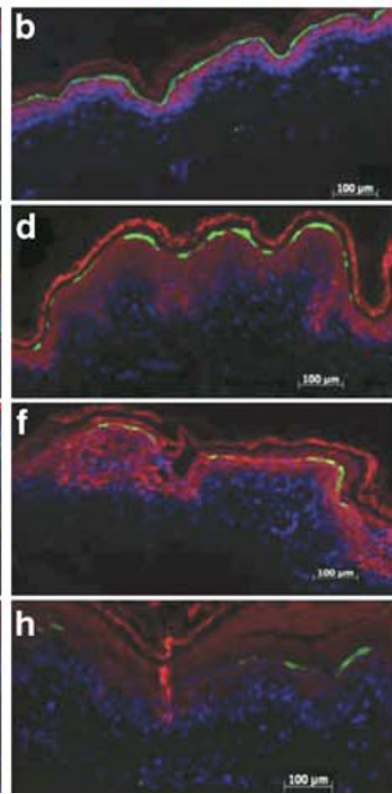

Organotypic Culture
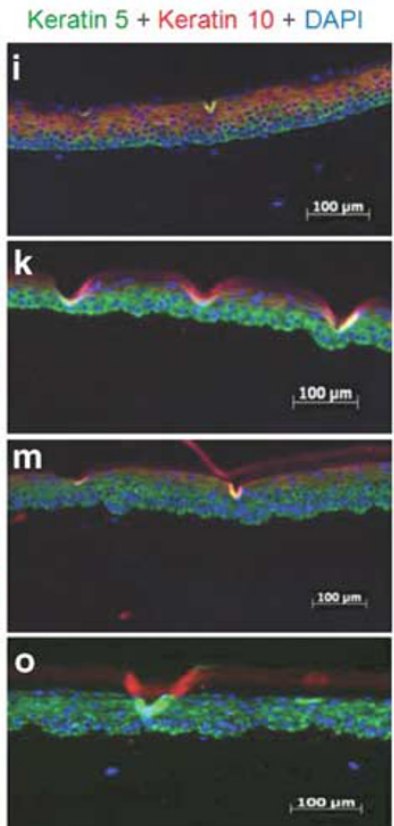

Loricrin+ Keratin $10+$ DAPI

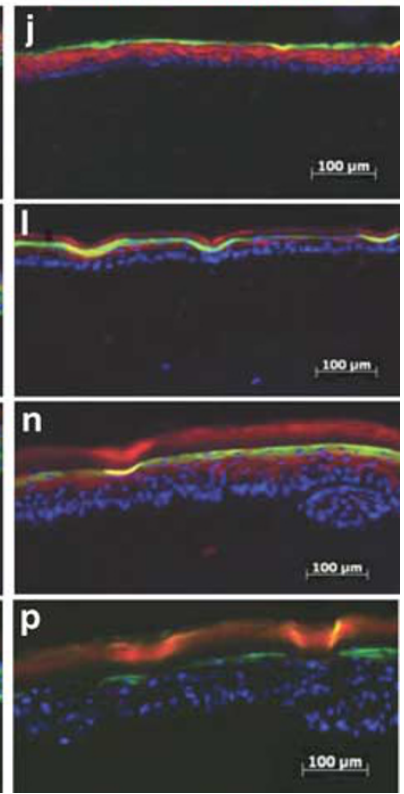

Figure 4 Differentiation of keratinocytes in epidermis of HESCs and OTCs. Expression of K5 was detected in the ex vivo culture and organotypic culture along the time course of culture. Although $\mathrm{K} 5$ was expressed primarily near the basal layer in ex vivo culture, its expression was detected across multiple layers in the organotypic culture. K10 expression in the ex vivo culture was found until day 12 (g, h). However, K10 expression was only found at POD0 in the organotypic culture (i, $\mathbf{j}$, day 7 post lift). Expression of loricrin was detected both in the ex vivo culture and organotypic culture. The signal of loricrin in both models was diminished at day $12(\mathbf{g}, \mathbf{h}, \mathbf{0}, \mathbf{p})$. (a, $\mathbf{c}, \mathbf{e}$, and $\mathbf{g})$ HESC with K5 and K10 staining at day 0, 4, 8, and 12; (b, d, f, and $\mathbf{h})$ HESC with loricrin and K10 staining at day $0,4,8$, and 12; (i, $\mathbf{k}, \mathbf{m}$, and $\mathbf{o}$ ) OCT with K5 and K10 staining at day 0, 4, 8, and 12; (j, i, n, and p) OCT with loricrin and K10 staining at day $0,4,8$, and 12 . The length of the scale bar is $100 \mu \mathrm{m}$.

Reproduction of the collagen in the dermal layer, which occurs during wound repair, was addressed via collagen staining. The newly generated collagen was not detected at day 0 or day 2 (Figures 10a and b) post injury. However, a considerable amount of new collagen was detected at day 6 as seen in Figure 10c. The two areas with dark green color clearly suggest new collagen fibers, making the original incision sites easily differentiated from the surrounding areas, which appear to be a bright yellow/orange color. Less newly generated collagen was found at day 8 (Figure 10d) compared with day 6 (Figure 10c). By day 8, the regenerated dermis could not be easily differentiated from the surrounding noninjured tissue (Figure 9d).

Activation of keratinocytes, which is found in normal wound healing, was address by analyzing the expression of Ki-67. Immediate sampling after incisions did not show the difference of Ki-67 expression between wounded and unwounded areas (Figures 11a and b). Increase of Ki-67 positive keratinocytes was clearly observed in the wound bed at day 2 compared with unwounded HESCs (Figures 11c and d). The proliferation of keratinocytes in the wound bed remained upregulated at day 6 (Figures 11e and f). It was noted that the proliferating cells near the bed were distributed across multiple layers of the epidermis in contrast to unwounded HESCs in which the proliferating keratinocytes were primarily located along the basal layer. Activation of keratino- cytes in the wound bed started to be attenuated at day 8 post wounding (Figures $11 \mathrm{~g}$ and $\mathrm{h}$ ).

Activation of keratinocytes was also estimated by immunofluoresence of K6. Under normal culture conditions, the expression of K6 in HESC was slightly elevated along the time course of the culture. Expression of K6 was slightly increased before day 4 and decreased after day 4 during the skin culture (Figures 12a-e). Similarly, in the skin graft with incisional grids, the increase of $\mathrm{K} 6$ expression was also observed before day 4 during culture; however, there was no significant decrease of expression levels of K6 after 4 days post wounding (Figures $12 \mathrm{f}-\mathrm{j}$ ). By comparing the expression levels of K6 between the unwounded and wounded skin, we found that the expression of $\mathrm{K} 6$ was more induced in wounded skin than in unwounded skin at all time points. Although the expression level of K6 at day 4 in wounded skin was higher than any other time points, the biggest difference of K6 expression between the wounded and unwounded skin came at day 12 post wounding. Thus, analyses of incisional wounds in HESCs suggest that our ex vivo culture model behaves similar to the in vivo human skin.

\section{Wound Closure in the Organotypic Cultures}

The incisions made on the OTCs closed faster than those on HESCs. The epithelial gap could only be seen at day 0 and 2 post wounding (Figures 13a and b). Thereafter, the injured 

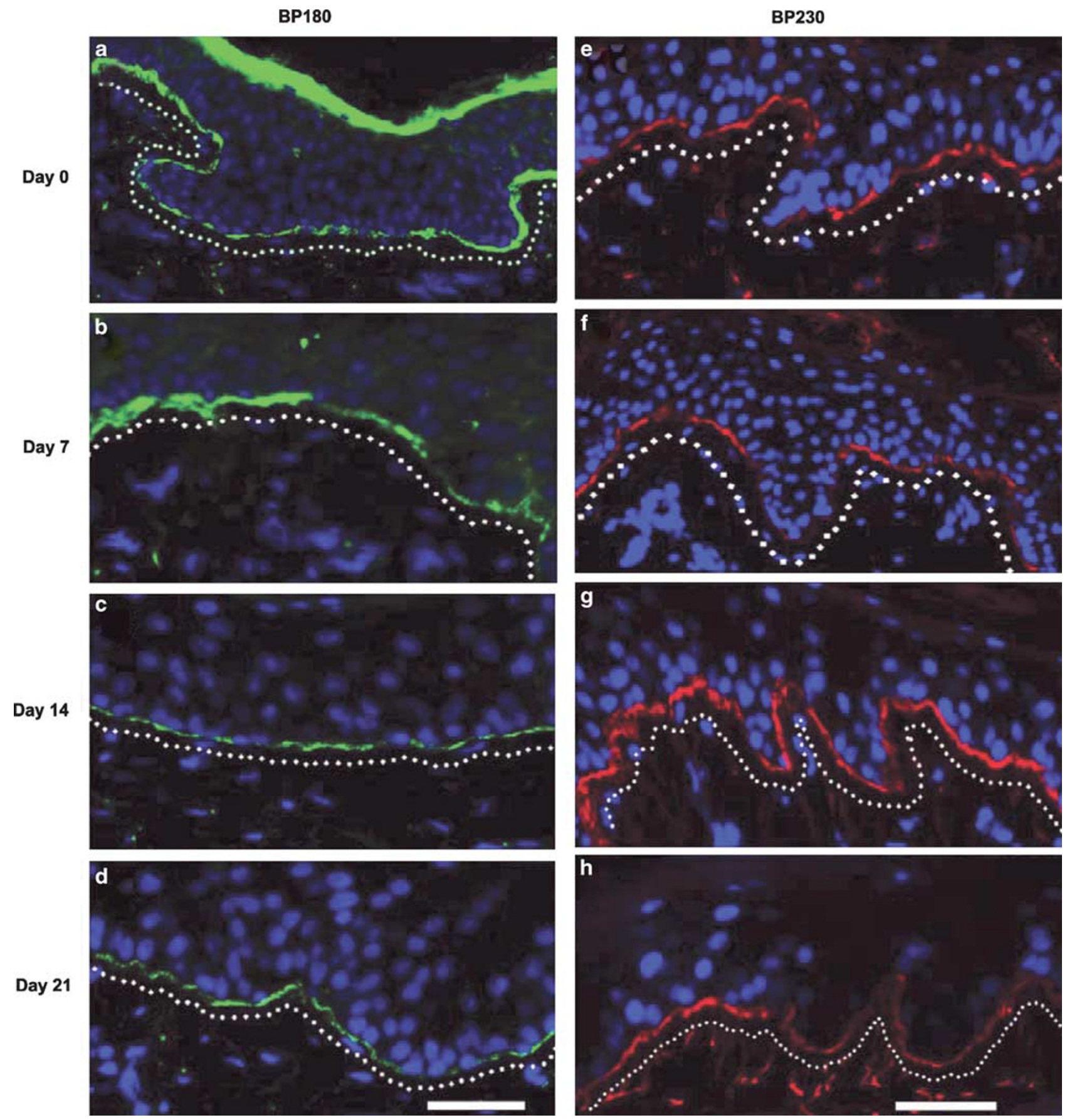

Figure 5 Immunofuorescence staining with hemidesmosome BP 180 (a-d, green) and BP 230 (e-h, red) in HESCs. The two bullous pemphigoid antigens were detected at different stages of skin cultures. The continuous distribution of BP 180 and BP 230 suggested the integrity of basal layer of the epidermis. The length of the scale bar is $100 \mu \mathrm{m}$.

sites could not be recognized (data not shown). The immunofluorescent staining with Ki-67 and K6 did not show any difference between the injured and non-injured tissues (Figures $13 \mathrm{c}-\mathrm{j}$ ). There were also no signs of remodeling of the dermal matrix as artificial collagens were used in the organotypic cultures. These differences, combined with the presence of k5 staining basal cells in all layers, (Figure 4) emphasize that in the organotypic cultures, keratinocytes are not normally differentiated in the progression of the basal cell layer through the stratum corneum layer, and appear to be very actively proliferating even without injury.

\section{DISCUSSION}

The development of the HESC allows investigators to study human skin in in-vitro conditions. Skin explants have been applied in many studies but a thorough examination of the 
a

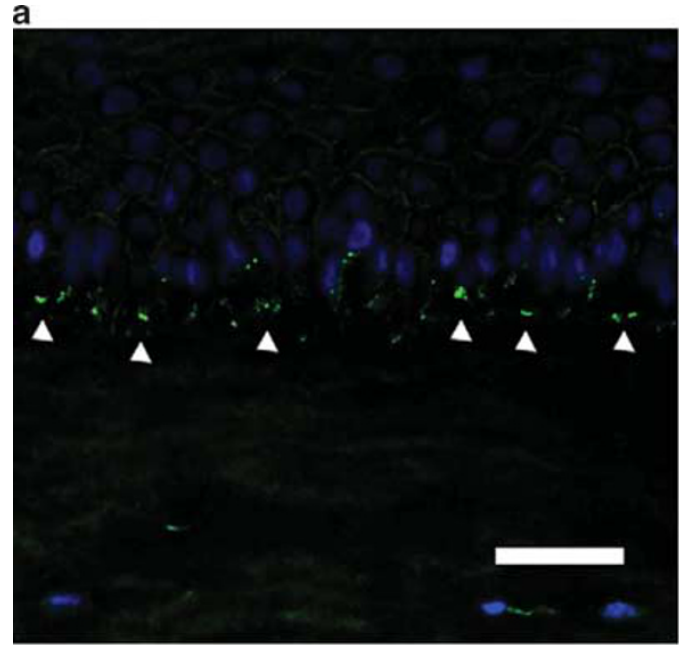

b

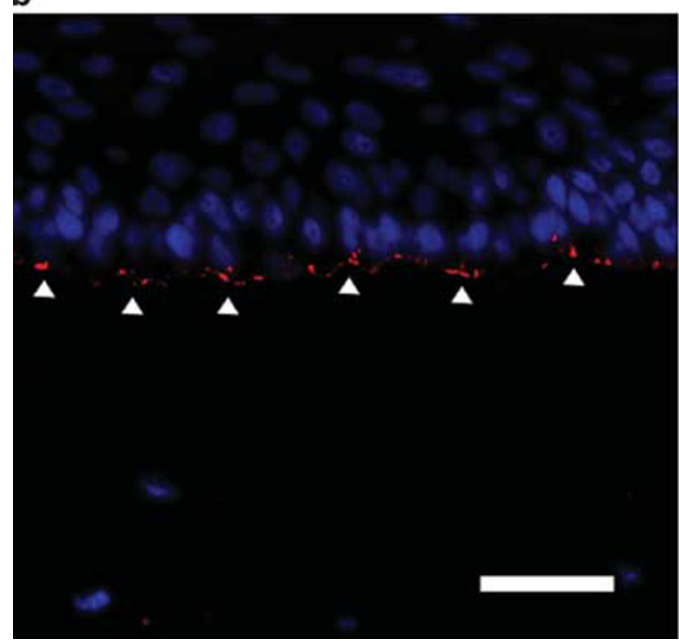

Figure 6 Immunofuorescence staining with BP 180 (a, green) and BP 230 (b, red) in OTCs. The signals for the two hemidesmosome components were observed between the epidermis and dermis with discontinuous distribution. The length of the scale bar is $100 \mu \mathrm{m}$.

viability of the skin and the fidelity of the behavior of skin to injury has not previously been undertaken. In our model, we employed a series of new methods to overcome the limitations that were noticed in earlier studies, such as the small area of the specimen and lack of tension during the skin culture. ${ }^{27-29}$ Our model also carries some unique characters, which have never been reported in previous studies. For example, in our system, the normal morphology of epidermis could be maintained for at least 12 days; the basement membrane was intact after 21 days of culture; keratinocyte proliferated up to 12 days, whereas apoptosis started after 6 days culture. Moreover, the epidermis re-epithelialization, and dermal collagen reproduction and remodeling were all observed during wound healing. All these functions strongly suggest that our HESC behaves similar to human skin in vivo and can be used as a substitute for human subjects in wound repair studies. All these advantages benefit from the novel procedures applied in this model.
The human skin explant in this model was fixed by sutures around each sample providing an even level of tension across the surface, to a level comparable to in vivo resting tension. Also, only a part of the dermis was submerged by medium allowing the epidermis to be exposed to an air interface similar to in vivo skin. This induced the keratinocytes in surface layer to form the stratum corneum, which is the water barrier layer in skin. ${ }^{30,31}$ In our model, the stratum corneum layer was clearly observed at different stages of culture. Compared with the fresh skin sample, the layers of stratum corneum were well conserved and even increased, (Figure 3) which suggested that our culture system provided a representative air-liquid interface environment for the epidermis. The well-maintained stratum corneum also reduced the water loss from the epidermis so that the hydration conditions of the keratinocytes were relatively stable. Morphologically, the keratinocytes in HESC did not show differences compared with fresh human skin (Figures 3-5). The keratinocytes in HESC were still able to proliferate until day 12 (Figure 7). However, our ex vivo culture model had a considerable number of cells apoptosis beginning about 1 week after culture (Figure 8). As shown in the keratinocytes differentiation with keratins and loricrin staining, the ex vivo cultured skin could keep the basal layer, spinous layer, and stratum corneum for at least 8 days (Figure 4). This, combined with the data on proliferation, and preservation of the basement membrane, suggests that the behavior of the skin to various perturbations would reasonably mimic in vivo situations for up to 6 days. Longterm culture of HESCs, of over 12 days, caused severe destruction of the epidermis. Differentiation of the keratinocytes is known to require the involvement of many factors. The maintenance of the differentiated cells suggests that our HESC model was surrounded by proper environment.

The optimal culture time in each skin culture model may vary. For instance, the study of hair-follicles physiology allowed the investigators to use skin explants cultured for more than 2 weeks $^{32}$ in the investigation of primary cultured hair follicle-derived cells. In addition, the skin was ex vivo cultured for more than 8 weeks as the investigators used the skin as a matrix to sustain growth of follicle-derived cells. ${ }^{27}$ The method used in our study kept the HESCs for about 2 weeks without severe modification of the physiology and histology of the keratinocytes (Figures 3-5). However, changes in apoptosis suggest that studies extending beyond 1 week are more difficult to correlate to in vivo behavior (Figure 8). This observation is consistent with the previous investigation done by Steinstraesser et $a l^{33}$ which suggested that it was difficult to culture the human skin ex vivo for more than 2 weeks as the physiologic turnover of healthy human epidermis is about 14 days.

Two weeks ex vivo culture allows the study of wound healing with this model as the re-epithelialization time of the incisions in our study was about 6 days (Figures 9 and 11). Previously, wounding area re-epithelialization assay was 

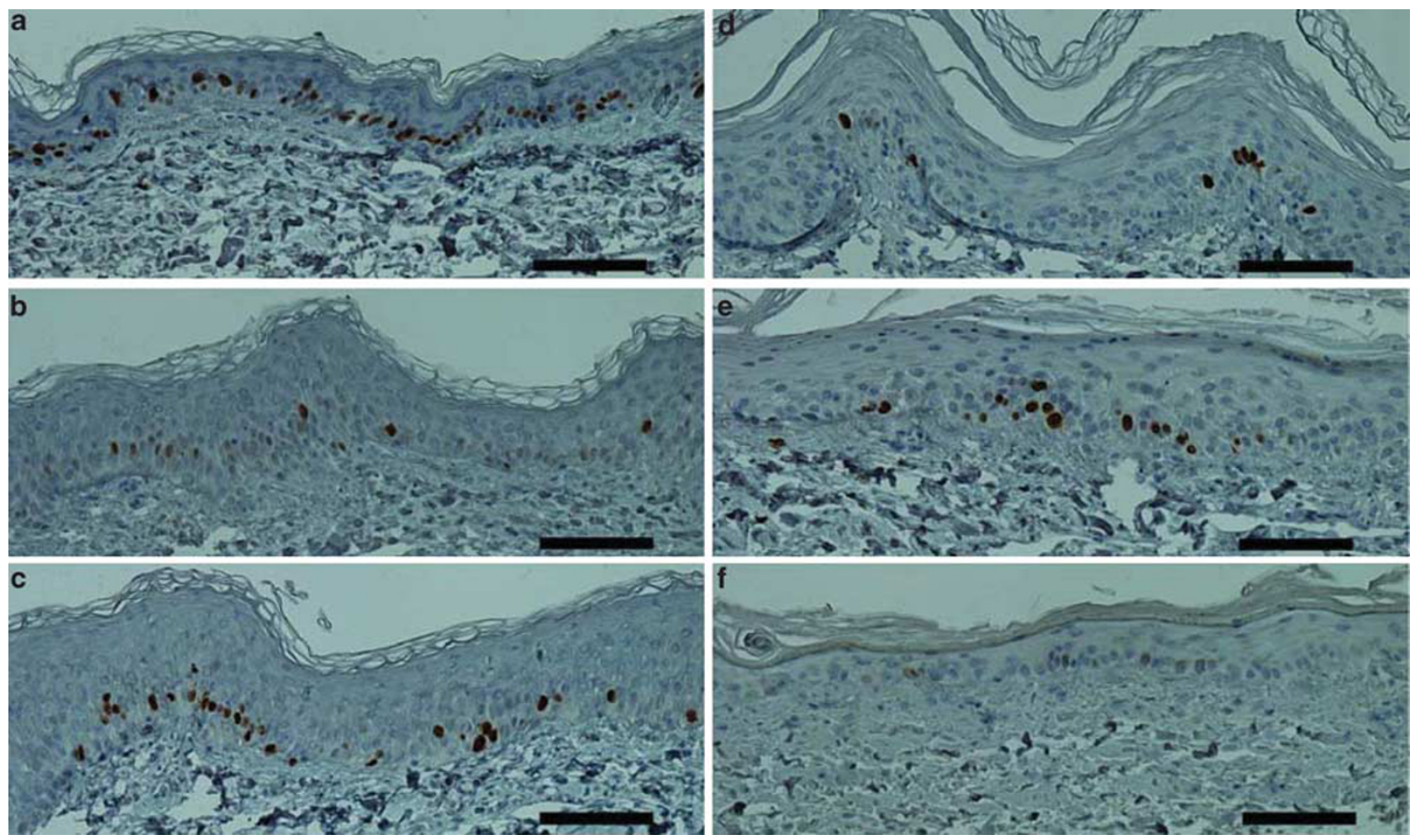

Figure 7 Identification of cell proliferation in HESCs with Ki67 IHC. The maximum number of proliferating keratinocytes were shown in a fresh skin sample (a) and proliferating cells were also seen from day 2 through day 12 (b-e are day 2, 4, 6, and 12, respectively) post skin grafting. There were no cells at day 14 (f) showing positive proliferation signal. The length of the scale bars is $50 \mu \mathrm{m}$.
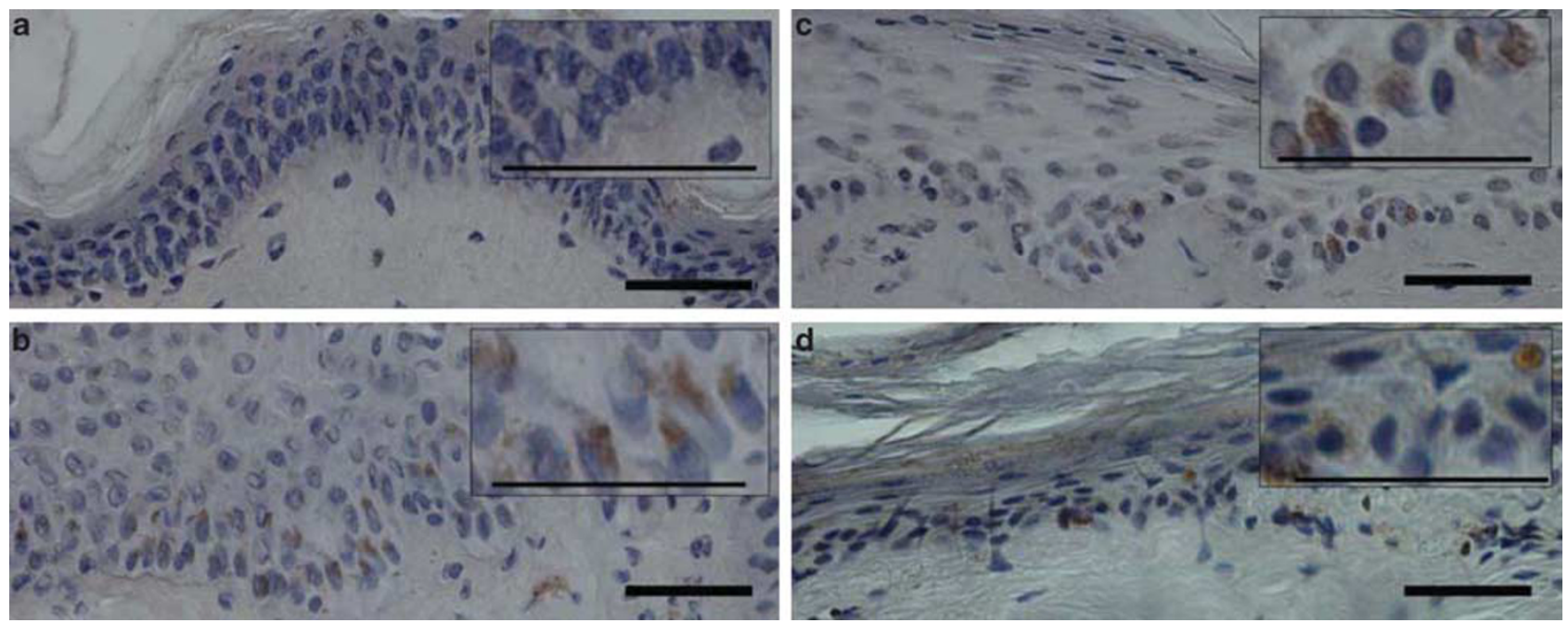

Figure 8 Keratinocyte apoptosis analysis on HESCs. Minimal cell apoptosis was observed at day 0 (a). Increased apoptotic cells were observed at day 6 (b) and day 12 (c), whereas only a few apoptotic keratinocytes were seen at day 14 (d) post skin grafting. Apoptotic keratinocytes were analyzed with the TUNEL system and apoptoic cells were stained brown. The length of the scale bars is $50 \mu \mathrm{m}$.

performed earlier on a fetal in vitro explant model. In that model, the epithelialization was observed between day 4 and 7 post wounding with a significant hypertrophic epidermis around the wounding area. ${ }^{15}$ The observation in fetal explants was very similar to our results in adult skin, although different methods were used in the two studies to create and maintain the skin explants.
In our HESC model, the incisional wounds successfully activate keratinocyte proliferation during healing (Figure 12). However, the proliferation rate of keratinocytes near basal layer in normal OTC was almost $100 \%$, and thus could not be further stimulated by injury (Figure 13). The epithelial gap of the OTC was filled rapidly post wounding. We were not able to identify the wounding site with proliferation markers after 

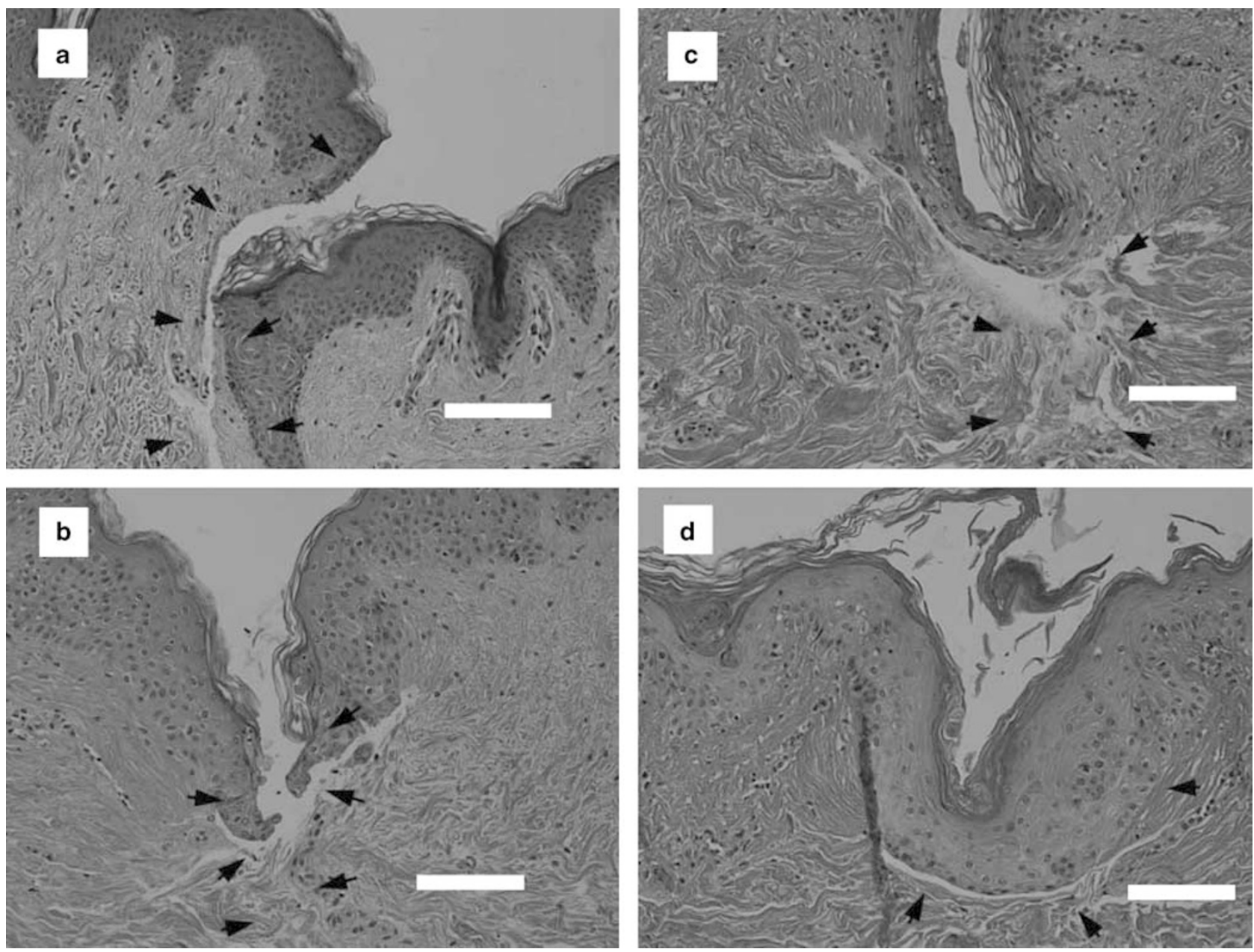

Figure 9 Re-epithelialization of incisions on HESCs. The incision was clearly observed immediately after wounding (a). The break of epithelium was still found at day 2 post wounding, while initial migration of keratinocytes in the edge of the wound towards closure was noticed (b). A fully re-epithelialized epidermis was observed at day 6 post wounding (c). At day 8 post wounding, there was a hyperplasia at the original wounding area (d). Black arrows indicated the original incision areas. The length of the scale bars is $50 \mu \mathrm{m}$.

the wound was healed (data not shown). These data, combined with the observations of abnormal differentiation in the OTCs, suggest that the OTCs have limitations for the wound healing study.

Our results were also reinforced by the observation of the induction of $\mathrm{K} 6$ during the wound healing process. In vivo experiments previously have demonstrated that expression of K6 persisted as keratinocytes migrated towards the wounding site and this process was reversible upon wound closure. ${ }^{34}$ In our study, persistent elevations in expression levels of K6 from day 2 to day 12 were detected in HESCs (Figure 12). In another experiment, the keratinocyte proliferation rate was also rebooted by incisions in HESC (Figure 11). The re-epithelialization and activation of $\mathrm{K} 6$ in skin ex vivo cultures after wounding, supports the clinical relevance of our model (Figures 9, 11, and 12).

Besides the well-maintained structures of epidermis and dermis in our HESC model, it is noticeable that another important constituent of human skin, the basement membrane, was also well preserved during the culture. The basement membrane in skin serves as a multifunctional structure separating epithelium from mesenchyme and provides a firm support for the germinative basal cell layer. ${ }^{35,36}$ It regulates the phenotype and differentiation of various types of cells ${ }^{37}$ and has been identified to be involved in many pathological processes, such as wound healing and tumor cell invasion. ${ }^{38}$ Many molecules with multiple roles have been identified from the basement membrane including laminins, type IV and VII collagens, nidogen, perlecan, BP180, and BP230. BP180 and 230 are two important protein components of basement membrane and constitutively produced by keratinocytes, while anchoring basal layer keratinocytes to the basement membrane through DEJ. Well-maintained human skin with good integrity has continuously distributed BP180 and 230 within the basement membrane. The lack of these BP180 and 230 antigen proteins results in insecure attachment of the epidermis to the dermis. The HESC used in our study demonstrated persistent high density of BP180 and 230 in the basement membrane area (Figure 5). In contrast, the 

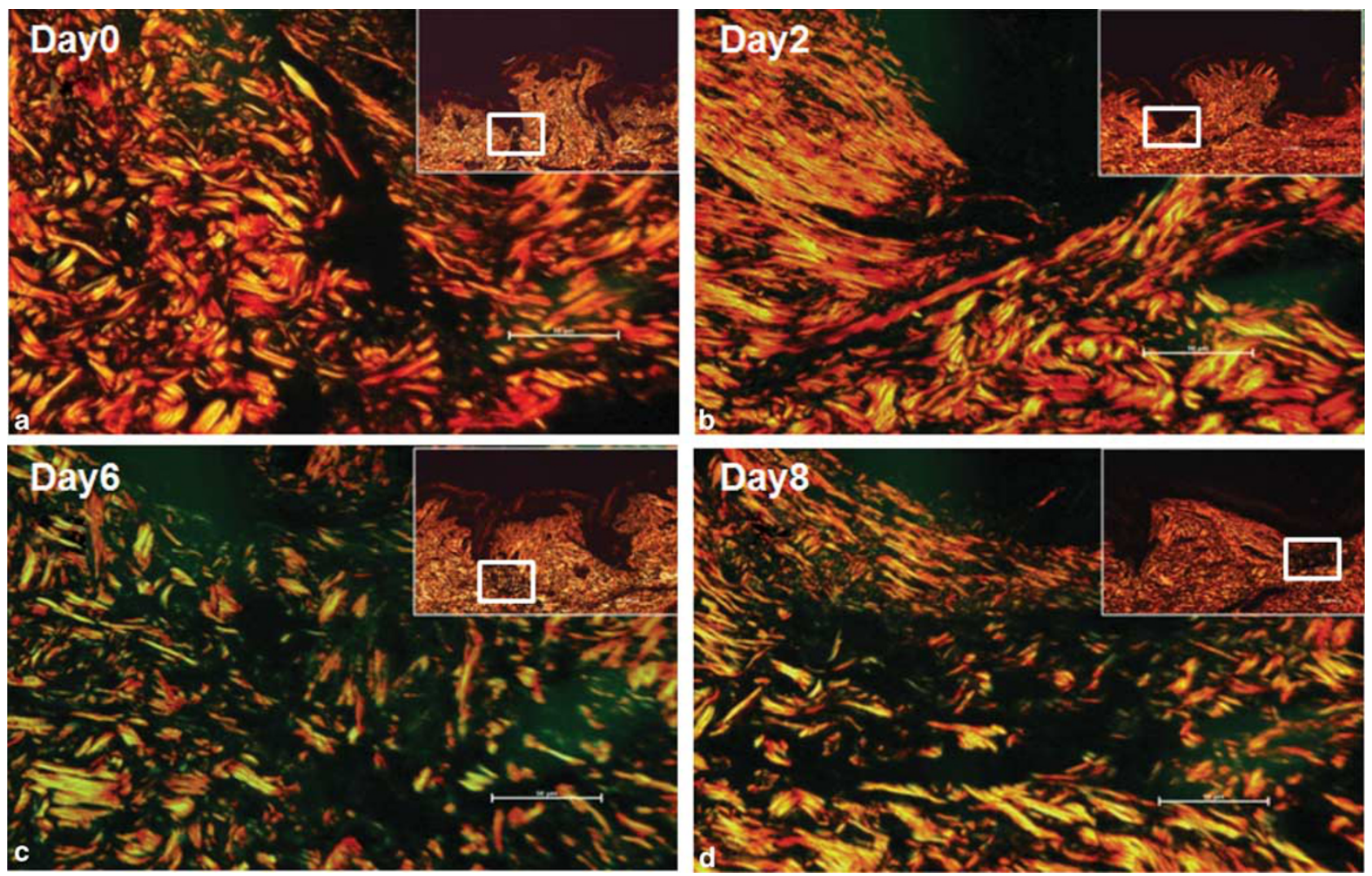

Figure 10 Picrosirius red staining to detect the generation of collagen post wounding in HESCs. The staining of collagen at day 0 post wounding showed strong yellow/orange color due to lack of newly generated collagen (a). Similarly, there was no detectable new collagen identified by picoririus red by day 2 post wounding (b). The newly produced collagen was found around wounding areas at day 6 (c) and day 8 (d) post wounding as indicated by dark green color. Sections with lower magnification were shown in inlets. The length of the scale bars is $50 \mu \mathrm{m}$.

skin obtained through organotypic culture demonstrated a discontinuous spotty staining for BP180 and 230 (Figure 6). Similar results were observed in the study performed by Smola $e t ~ a l,{ }^{38}$ which showed that the formation of basement membrane was incomplete 3 weeks after the organotypic culture was transplanted to a nude mouse. However, under in vitro culture conditions, the organotypic raft culture demonstrated degradation to the epidermis layer within 2 weeks, and during that time period the basement membrane was not reconstituted. The lack of a DEJ is a limitation of the OTCs, compared with the ex vivo model. Because the epidermis was so poorly attached to the underlying dermis, any attempt at incision resulted in shearing off a sheet of epidermal cells, rather than a precise incision. In addition, compared with the incisions in our HESCs, which left clear markers in the dermis when breaking the collagen fibers, the incisions on the organotypic culture barely left any markers in collagen matrix as this jelly-like material could be easily recovered. As a consequence it was impossible to detect the site of the incision after re-epithelization in OTCs. With an intact basement membrane, the HESC model has more reliability in studies of wound healing, especially for keratinocyte differentiation and keratinocyte-fibroblast interactions, which are affected by the basement membrane.
It is interesting that collagen production was observed during the healing of incisional wounds in the skin culture. This suggests, after days of ex vivo culture, the fibroblasts within the dermis are still active. The reconstruction of collagen in wounded areas appeared to lag behind the re-epithelialization of the epidermis. Although the collagen reconstruction during wound repair is a key event leading to scar formation and wound contraction, it is difficult to study this remodeling phase with current in vitro skin models. A variety of materials have been used to build dermal substitutes, such as collagen gels, ${ }^{39}$ collagen sponges, ${ }^{40}$ and polyglactin mesh. ${ }^{41,42}$ However, the re-organization of the matrix has not been well characterized. In our model, the generation of new collagen fibers was noted 6 days post wounding (Figure 10), which suggested that our HESC model could be potentially used in scar studies. It will be worthwhile to find a way to delay the turnover time of the tissue so that we can utilize this model to monitor the process of scar formation.

Also, in our HESC model, some other types of cells were well preserved beside keratinocytes and fibroblasts. Based on the immunofluorescence staining with cell markers, we found alpha smooth muscle actin ( $\alpha$-SMA), CD31, and CD34-positive cells (Supplementary Figure 1) after 10 days in culture. In contrast, the signals of these three markers in organotypic cultures are 
negative as expected. It has been previously reported that in normal mouse or human skin, cells with $\alpha$-SMA, CD31, or CD34 markers were detected. The $\alpha$-SMA is a cell marker for myofibroblast, ${ }^{43}$ or endothelial cells derived from CD34-positive cord blood cells. ${ }^{44}$ The CD31 is primarily expressed in endothelial cells ${ }^{45}$ whereas CD34 is a marker for dendritic cells ${ }^{46}$ or some cells located in hair follicles. ${ }^{47}$ Although we do not have enough evidence to identify the cell type with a single cell marker, it is at least indicative of a diversity of the cell types in HESC model. This will be potentially useful in the study of the cell fate and cell-cell interactions during the wound repair.

In summary, the partial-thickness HESC model developed in this study maximized strengths of the in vitro model in the study of wound healing. The characteristics carried by natural
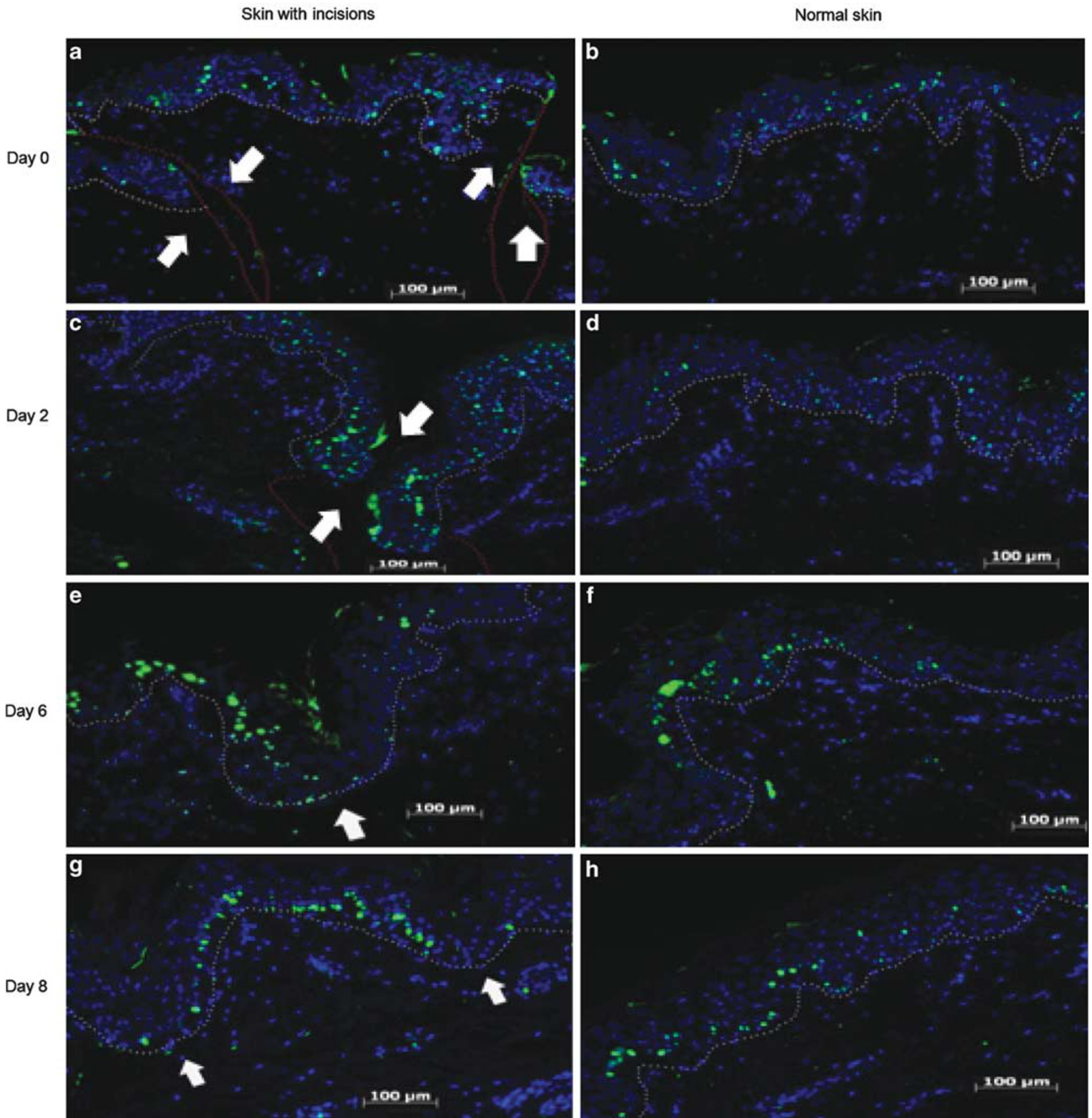

Figure 11 Activation of keratinocytes in the wound bed of HESCs. Ki67 was used as a marker for cell proliferation during wound healing. (a) HESC with incision at day 0 post wounding; (b) HESC without incision at day 0. More proliferating cells were observed near the wound bed at day 2 (c) and day 6 (e) compared with the unwounded area $(\mathbf{d}, \mathbf{f})$. At day 8 post wounding, there was no significant difference in the number of proliferating cells between wounded $(\mathbf{g})$ and unwounded (h) areas. The basement membrane was outlined by white dash lines. The arrows indicated the original incisions. The length of the scale bars is $100 \mu \mathrm{m}$. 


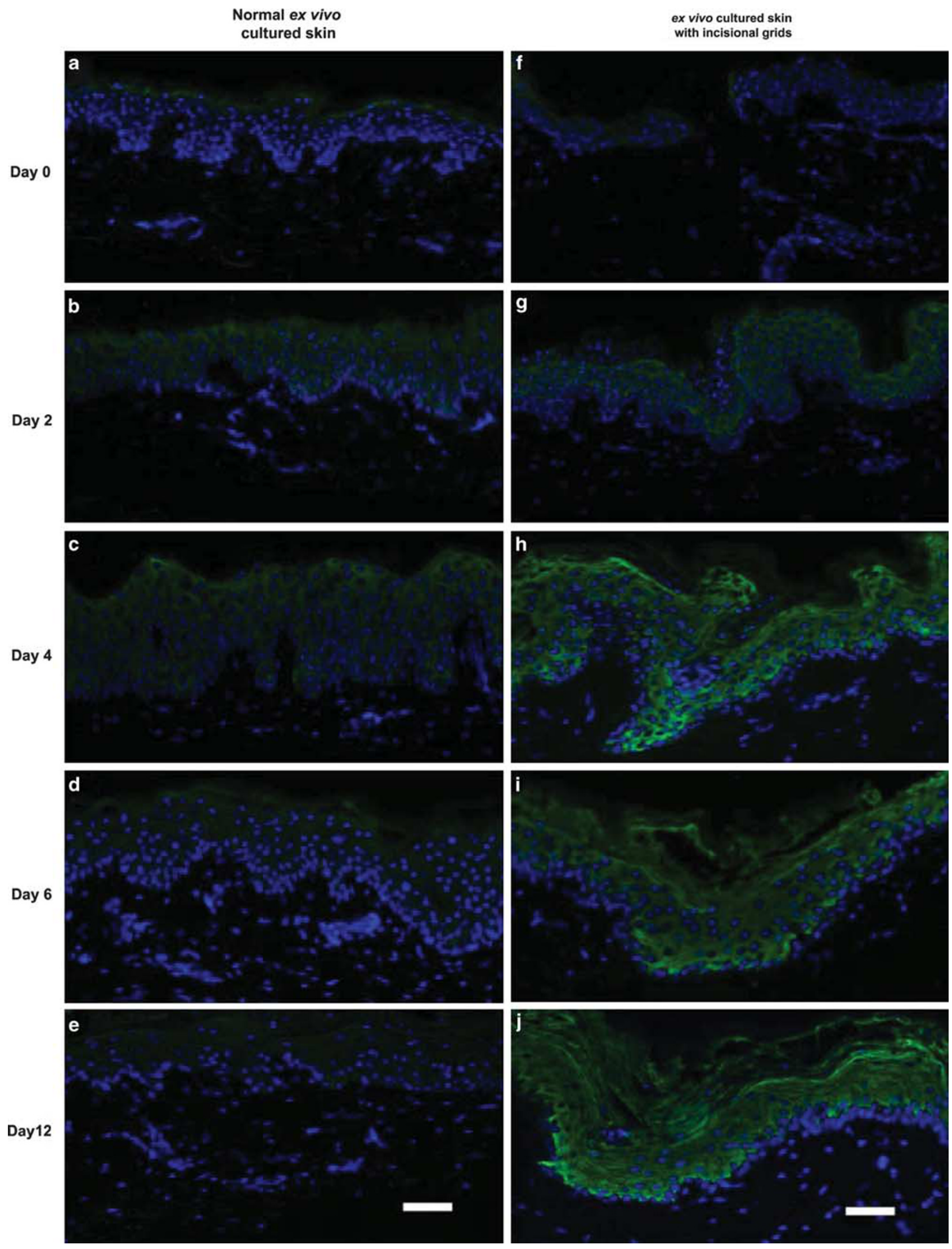


Day 0

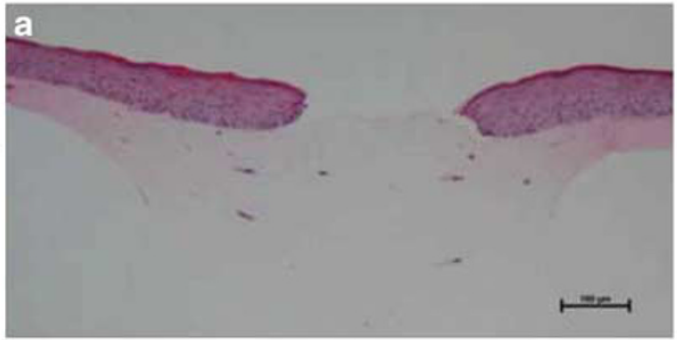

Wounded, H\&E

Wounded, Ki67 + DAPI
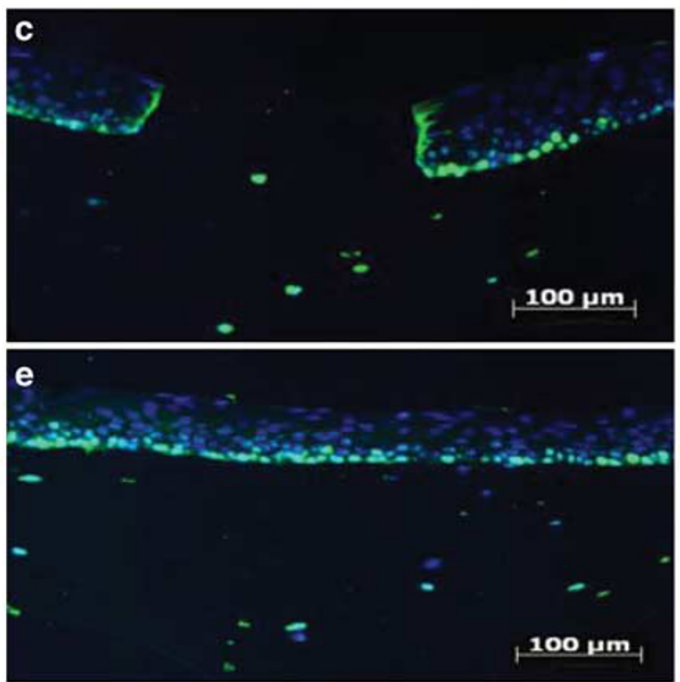

Normal, Ki67 + DAPI

Wounded, $K 6$ + DAPI

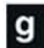

Normal, K6 + DAPI

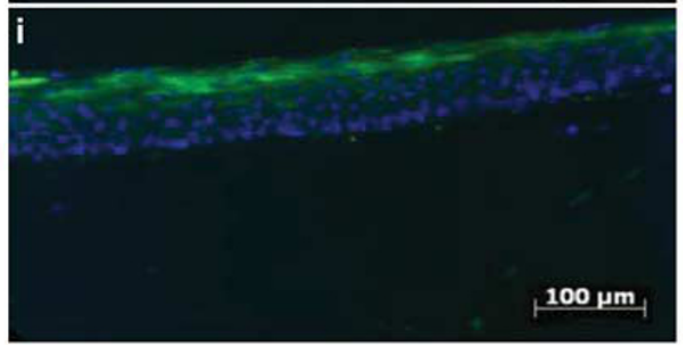

$100 \mu \mathrm{m}$

Day 2

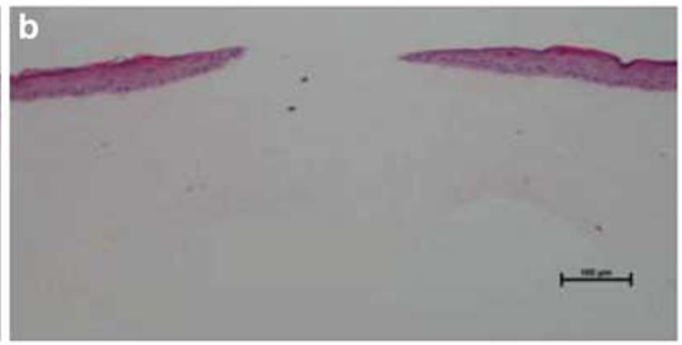

d
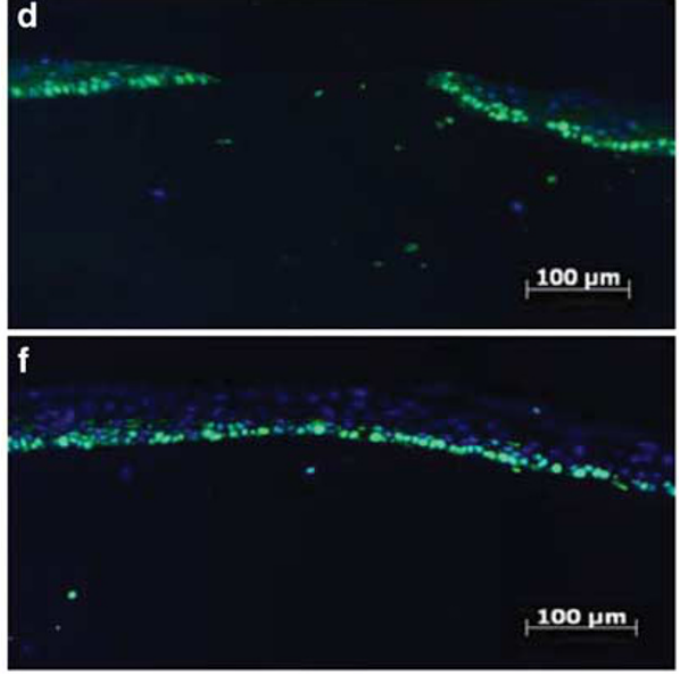

h

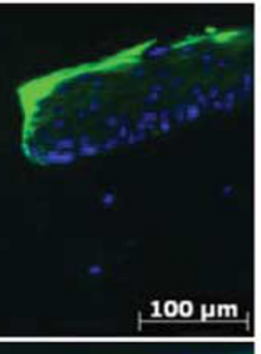

i

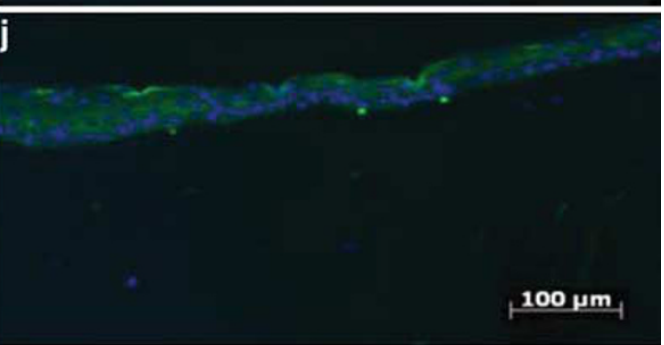

Figure 13 The healing of the incisions in OTCs. A faster healing rate was observed in the organotypic culture compared with the ex vivo culture model. The epithelial gaps were only seen at day 0 and day 2 post wounding (a, b). Expression levels of Ki-67 in wound beds at day 0 and day 2 (c, d) did not show significant difference than unwounded area at day 0 and day $2(\mathbf{e}$, f). Similarly, there was no obvious difference between the incision ( $\mathbf{g}, \mathbf{h}$ ) area and normal area (i and $\mathbf{j}$ ) at day 0 and day 2 . The length of the scale bars is $100 \mu \mathrm{m}$.

Figure 12 Immunofluorescent staining for keratin 6 in HESCs with $(\mathbf{a}-\mathbf{e})$ or without incisional grids $(\mathbf{f}-\mathbf{j})$ on the surface. Expression of keratin 6 (k6) was slightly induced by the normal ex vivo culture of the human skin, whereas its expression was significantly stimulated by incisional wounding. Expression levels of $k 6$ reached the peak at day 4 (c) post wounding and decreased after this time point. Expression of k6 in the skin with incisional grids was also significantly induced from day 4 (h) whereas high levels of k6 expression lasted till the end of the skin culture. The length of the scale bars is $100 \mu \mathrm{m}$. 
human skin were well preserved in the model. Of significance, healing of superficial wounds was observed with epithelial differentiation and proliferation in the epidermis, and a new collagen deposition in the dermis. Thus, our HESC model has several advantages as a model system to facilitate study of in vivo wound healing.

Supplementary Information accompanies the paper on the Laboratory Investigation website (http://www.laboratoryinvestigation.org)

\section{ACKNOWLEDGEMENTS}

We would like to thank Dr Jonathan Jones for the BP180 \& BP230 antibodies, and Matthew Geringer and Dr Madeleine Gust for their critical review of the manuscript. This work was supported by internal funding from Division of Plastic and Reconstructive Surgery, Northwestern University, Feinberg School of Medicine.

\section{DISCLOSURE/CONFLICT OF INTEREST}

The authors declare no conflict of interest.

1. Gottrup F, Agren MS, Karlsmark T. Models for use in wound healing research: a survey focusing on in vitro and in vivo adult soft tissue. Wound Repair Regen 2000;8:83-96.

2. Grose $R$, Werner $S$. Wound healing studies in transgenic and knockout mice. A review. Methods Mol Med 2003;78:191-216.

3. Dorsett-Martin WA. Rat models of skin wound healing: a review. Wound Repair Regen 2004;12:591-599.

4. Ahn ST, Mustoe TA. Effects of ischemia on ulcer wound healing: a new model in the rabbit ear. Ann Plast Surg 1990;24:17-23.

5. Saatman RA, Carlton WW, Hubben K, et al. A wound healing study of chlorhexidine digluconate in guinea pigs. Fundam Appl Toxicol 1986;6:1-6.

6. Steinstraesser $L$, Sorkin $M$, Niederbichler $A D$, et al. A novel human skin chamber model to study wound infection ex vivo. Arch Dermatol Res 2010;302:357-365.

7. Nasca MR, $\mathrm{O}^{\prime}$ Toole EA, Palicharla $\mathrm{P}$, et al. Thalidomide increases human keratinocyte migration and proliferation. J Invest Dermatol 1999;113: 720-724.

8. Tao H, Berno AJ, Cox DR, et al. In vitro human keratinocyte migration rates are associated with SNPs in the KRT1 interval. PLoS One 2007;2:e697.

9. Tandara AA, Mustoe TA. MMP- and TIMP-secretion by human cutaneous keratinocytes and fibroblasts-impact of coculture and hydration. J Plast Reconstr Aesthet Surg 2011;64:108-116.

10. Oberringer $M$, Meins $C$, Bubel $M$, et al. A new in vitro wound model based on the co-culture of human dermal microvascular endothelial cells and human dermal fibroblasts. Biol Cell 2007:99:197-207.

11. Garlick JA, Taichman LB. Fate of human keratinocytes during reepithelialization in an organotypic culture model. Lab Invest 1994;70:916-924.

12. Maas-Szabowski N, Stark HJ, Fusenig NE. Keratinocyte growth regulation in defined organotypic cultures through IL-1-induced keratinocyte growth factor expression in resting fibroblasts. J Invest Dermatol 2000;114:1075-1084.

13. Peramo A, Marcelo CL, Goldstein SA, et al. Novel organotypic cultures of human skin explants with an implant-tissue biomaterial interface. Ann Biomed Eng 2009;37:401-409.

14. Greenwald DP, Gottlieb LJ, Mass DP, et al. Full-thickness skin wound explants in tissue culture: a mechanical evaluation of healing. Plast Reconstr Surg 1992;90:289-294.

15. Burd DA, Longaker MT, Adzick NS, et al. Fetal wound healing: an in vitro explant model. J Pediatr Surg 1990;25:898-901.

16. Kirkpatrick CJ, Krump-Konvalinkova V, Unger RE, et al. Tissue response and biomaterial integration: the efficacy of in vitro methods. Biomol Eng 2002;19:211-217.

17. Steinstraesser $L$, Hirsch $T$, Beller J, et al. Transient non-viral cutaneous gene delivery in burn wounds. J Gene Med 2007;9:949-955.
18. Gillery P, Bellon G, Coustry F, et al. Cultures of fibroblasts in fibrin lattices: models for the study of metabolic activities of the cells in physiological conditions. J Cell Physiol 1989;140:483-490.

19. Varani J, Kang S, Stoll S, et al. Human psoriatic skin in organ culture: comparison with normal skin exposed to exogenous growth factors and effects of an antibody to the EGF receptor. Pathobiology 1998;66:253-259.

20. Yoshinaga $Y$, Higaki $M$, Terajima $S$, et al. Detection of inflammatory cytokines in psoriatic skin. Arch Dermatol Res 1995;287:158-164.

21. Rambukkana A, Bos JD, Irik D, et al. In situ behavior of human Langerhans cells in skin organ culture. Lab Invest 1995;73:521-531.

22. Larregina AT, Watkins SC, Erdos G, et al. Direct transfection and activation of human cutaneous dendritic cells. Gene Ther 2001;8: 608-617.

23. Morelli A, Larregina A, Chuluyan E, et al. Functional expression and modulation of C5a receptor (CD88) on skin dendritic cells. Adv Exp Med Biol 1997;417:133-138.

24. Larregina AT, Morelli $A E$, Spencer $L A$, et al. Dermal-resident CD14+ cells differentiate into Langerhans cells. Nat Immunol 2001;2: 1151-1158.

25. Morelli AE, Rubin JP, Erdos G, et al. CD4+ T cell responses elicited by different subsets of human skin migratory dendritic cells. J Immunol 2005;175:7905-7915.

26. Seiler WO, Stahelin HB, Zolliker R, et al. Impaired migration of epidermal cells from decubitus ulcers in cell cultures. A cause of protracted wound healing? Am J Clin Pathol 1989;92:430-434.

27. Krugluger W, Rohrbacher W, Laciak K, et al. Reorganization of hair follicles in human skin organ culture induced by cultured human follicle-derived cells. Exp Dermatol 2005;14:580-585.

28. Tavakkol A, Varani J, Elder JT, et al. Maintenance of human skin in organ culture: role for insulin-like growth factor-1 receptor and epidermal growth factor receptor. Arch Dermatol Res 1999;291: 643-651.

29. Moll I, Houdek P, Schmidt $H$, et al. Characterization of epidermal wound healing in a human skin organ culture model: acceleration by transplanted keratinocytes. J Invest Dermatol 1998;111:251-258.

30. Prunieras $M$, Regnier M, Woodley D. Methods for cultivation of keratinocytes with an air-liquid interface. J Invest Dermatol 1983;81 (1 Suppl):28s-33s.

31. Regnier $\mathrm{M}$, Caron $\mathrm{D}$, Reichert $\mathrm{U}$, et al. Barrier function of human skin and human reconstructed epidermis. J Pharm Sci 1993;82:404-407.

32. Lu Z, Hasse $S$, Bodo $E$, et al. Towards the development of a simplified long-term organ culture method for human scalp skin and its appendages under serum-free conditions. Exp Dermatol 2007;16: 37-44.

33. Steinstraesser L, Rittig A, Gevers K, et al. A human full-skin culture system for interventional studies. Eplasty 2009;9:e5.

34. Takahashi K, Yan B, Yamanishi K, et al. The two functional keratin 6 genes of mouse are differentially regulated and evolved independently from their human orthologs. Genomics 1998;53: 170-183.

35. Timpl R. Structure and biological activity of basement membrane proteins. Eur J Biochem 1989;180:487-502.

36. Breitkreutz D, Mirancea N, Schmidt C, et al. Inhibition of basement membrane formation by a nidogen-binding laminin gamma1-chain fragment in human skin-organotypic cocultures. J Cell Sci 2004;117(Part 12):2611-2622.

37. Lin CQ, Bissell MJ. Multi-faceted regulation of cell differentiation by extracellular matrix. FASEB J 1993;7:737-743.

38. Smola H, Stark HJ, Thiekotter $G$, et al. Dynamics of basement membrane formation by keratinocyte-fibroblast interactions in organotypic skin culture. Exp Cell Res 1998;239:399-410.

39. Compton CC, Butler CE, Yannas IV, et al. Organized skin structure is regenerated in vivo from collagen-GAG matrices seeded with autologous keratinocytes. J Invest Dermatol 1998;110:908-916.

40. Lamme EN, Van Leeuwen RT, Brandsma K, et al. Higher numbers of autologous fibroblasts in an artificial dermal substitute improve tissue regeneration and modulate scar tissue formation. J Pathol 2000;190: 595-603.

41. Kremer M, Lang E, Berger AC. Evaluation of dermal-epidermal skin equivalents ('composite-skin') of human keratinocytes in a collagenglycosaminoglycan matrix (integra artificial skin). $\mathrm{Br} J$ Plast Surg 2000;53:459-465. 
42. Waymack P, Duff RG, Sabolinski M. The effect of a tissue engineered bilayered living skin analog, over meshed split-thickness autografts on the healing of excised burn wounds. The Apligraf Burn Study Group. Burns 2000;26:609-619.

43. Wang J, Chen $\mathrm{H}$, Seth $\mathrm{A}$, et al. Mechanical force regulation of myofibroblast differentiation in cardiac fibroblasts. Am J Physiol Heart Circ Physiol 2003;285:H1871-H1881.

44. Lu X, Dunn J, Dickinson AM, et al. Smooth muscle alpha-actin expression in endothelial cells derived from CD34+ human cord blood cells. Stem Cells Dev 2004;13:521-527.
45. Miettinen $M$, Lindenmayer $A E$, Chaubal $A$. Endothelial cell markers CD31, CD34, and BNH9 antibody to $\mathrm{H}$ - and $\mathrm{Y}$-antigens-evaluation of their specificity and sensitivity in the diagnosis of vascular tumors and comparison with von Willebrand factor. Mod Pathol 1994;7:82-90.

46. Narvaez $D$, Kanitakis J, Faure $M$, et al. Immunohistochemical study of CD34-positive dendritic cells of human dermis. Am J Dermatopathol 1996;18:283-288.

47. Trempus CS, Morris RJ, Bortner CD, et al. Enrichment for living murine keratinocytes from the hair follicle bulge with the cell surface marker CD34. J Invest Dermatol 2003;120:501-511. 